\title{
Number of source patches required for population persistence in a source-sink metapopulation with explicit movement
}

\author{
Julien Arino $^{1}$, Nicolas Bajeux ${ }^{1,2}$, and Steve Kirkland ${ }^{1}$ \\ ${ }^{1}$ Department of Mathematics, University of Manitoba, Winnipeg, \\ Manitoba, Canada. \\ ${ }^{2}$ INRIA Sophia Antipolis, Sophia Antipolis, France.
}

Version of February 26, 2019

\begin{abstract}
We consider a simple metapopulation model with explicit movement of individuals between patches, in which each patch is either a source or a sink. We prove that, similarly to the case of patch-occupancy metapopulations with implicit movement, there exists a threshold number of source patches such that the population potentially becomes extinct below the threshold and established above the threshold. In the case where the matrix describing the movement of populations between spatial locations is irreducible, the result is global; further, assuming a complete mobility graph with equal movement rates, we use the principle of equitable partitions to obtain an explicit expression for the threshold. Brief numerical considerations follow.
\end{abstract}

\section{Introduction}

The problem of source-sink dynamics has been present in the ecology literature for some time, essentially originating with the pioneering work of Levins [29]. The idea is that species exist in habitats (often called patches) that can be of quite diverse qualities. These varying qualities mean that some of the habitats are favourable to species that live there, while others are not. The former are called sources, while the latter are sinks. Typically, sinks need an influx of individuals for a population to persist there. Of importance in this setting are the consequences for survival of the population as a whole. Source-sink dynamics have been investigated in a variety of contexts. For instance, [16] considers an extended prey-predator model inspired by marine ecosystems, [12] considers plant ecosystems. Refer to $[11,17,33]$ and references within for more detail. 
From a theoretical point of view, the question of heterogeneous landscapes has been widely studied in order to characterise the effect of colonisation and extinction rates of patches on the persistence of metapopulations; see [17]. These studies are based on the Levins model, in which the rate of change of the number of extant local populations is explicitly described. Levins-type models are typically patch occupancy models: state variables count the number (or proportion) of patches that are occupied by the species of interest. In these models, movement is implicit: spatial coupling is described through (typically) nonlinear terms accounting for the effect of populations at other spatial locations on the local population. In the case of such models, [17] study a variety of cases. They prove in particular that there is a threshold ratio of good to bad quality patches, i.e., a fraction of suitable patches, such that the overall population persists above the threshold and becomes extinct below.

Another type of metapopulation models are those with explicit coupling. Contrary to those models with implicit coupling, they describe the actual (explicit) movement of individuals between spatial locations [20,28] and are thus easier to parametrise in instances where movement data is available [5]; the abundance of both human and animal movement data available currently has made these models popular. Theoretical work on source-sink metapopulation systems with explicit movement is less advanced and has proceeded mainly along two axes. Low dimensional cases of two or three patches have been studied in great detail; see, e.g., $[4,23]$. On the other hand, large dimensional versions with $N$ patches have typically been considered numerically; see, e.g., [38]. Analytical work on a system of $N$ patches is carried out in [2] and [31]; in the latter, patches have prey-predator models. Closely related work in [37] considers a system comparable to the one here but without a focus on source-sink dynamics, i.e., patches are allowed to have somewhat individual dynamics but there is no requirement for some to be sources and others to be sinks.

In the present paper, similar results to those of [17] in the patch-occupancy with implicit movement case are obtained for the explicit movement case. Here, we explore an explicit model to highlight how the the threshold between source and sink patches is impacted by local populations (especially intrinsic growth rates) and movement between patches (migration matrix).

The present work follows [35], where a simple metapopulation model with explicit movement for source-sink dynamics on $N$ patches is considered for a single species. It is established numerically in [35] that there exists a threshold number of source patches in the system below which the system goes to a state with no population and above which the population becomes endemic. The model of [35] considers that movement is dependent on pressure, i.e., the direction of movement can change as a function of the difference in suitability of connected locations. This is in the same vein as, for instance, [34], where movement is a function of habitat quality.

We confirm analytically the result of [35] in a simplified setting with no dependence of movement on crowdedness. This simplification allows us to highlight the most important component underlying the process at hand: the relative number of source and sink patches determines the possibility for the global 
coupled system to sustain a population. In the case of a strongly connected movement digraph with movement equal whenever it happens, we use the principle of equitable partitions to derive an explicit formula for the threshold. Brief numerical considerations follow the analytical work. Relationship with existing work in the context of patch occupancy model is highlighted in Section 5.

\section{The model}

We consider a simplification of the model of [35]. Assume that there are $N>0$ patches, with $S \geq 0$ source patches and $N-S \geq 0$ sink patches. For simplicity, we assume that patches are ordered with all source patches first. While there is no real loss of generality in that assumption, we emphasise here that the assumption effectively specifies that, when changing from a model with $S$ source patches to a model with $S+1$ source patches, all of the former source patches are retained in the new model with $S+1$ source patches. Example 1 below points out another feature associated with our assumption on the ordering.

The model takes the form

$$
P_{i}^{\prime}=P_{i} g_{i}\left(P_{i}\right)+\sum_{j=1}^{N} m_{i j} P_{j}, \quad i=1, \ldots, N,
$$

where, in source patches, the per capita growth rate is

$$
g_{i}\left(P_{i}\right)=r_{i}\left(1-\frac{P_{i}}{K_{i}}\right), \quad i=1, \ldots, S
$$

and in sink patches, it is

$$
g_{i}\left(P_{i}\right)=-r_{i}, \quad i=S+1, \ldots, N,
$$

where $r_{i}>0$ for $i=1, \ldots, N$ and $K_{i}>0$ for $i=1, \ldots, S$. The model is considered with initial conditions $P_{i}(0) \geq 0$ for all $i=1, \ldots, N$. To avoid a trivial problem, it is further assumed that $\sum_{i=1}^{N} P_{i}(0)>0$. The parameter $r_{i}>0$ is both the growth rate in the case of source patches and the death rate in the case of sinks; $K_{i}>0$ is the carrying capacity of source patches. The movement rates $m_{i j}$ are nonnegative for $i \neq j$ and represent the rate at which individuals in patch $j$ move to patch $i$, with $m_{i j}=0$ if there is no direct movement from $j$ to $i$. The term $m_{i i}$ accounts for movement out of patch $i=1, \ldots, N$ and takes the form

$$
m_{i i}=-\sum_{j=1}^{N} m_{j i} .
$$

This allows us to write movement in the compact form in (1a). It also is useful to write the system in matrix form. The general form is

$$
\mathbf{P}^{\prime}=\mathbf{G}(\mathbf{P}) \mathbf{P}+\mathcal{M} \mathbf{P},
$$


where $\mathbf{P}=\left(P_{1}, \ldots, P_{N}\right)^{T}$,

$$
\mathbf{G}(\mathbf{P})=\operatorname{diag}\left(g_{1}\left(P_{1}\right), \ldots, g_{N}\left(P_{N}\right)\right)
$$

and

$$
\mathcal{M}=\left(\begin{array}{cccc}
-\sum_{j=1}^{N} m_{j 1} & m_{12} & \cdots & m_{1 N} \\
m_{21} & -\sum_{j=1}^{N} m_{j 2} & \cdots & m_{2 N} \\
& & & \\
m_{N 1} & m_{N 2} & \cdots & -\sum_{j=1}^{N} m_{j N}
\end{array}\right) .
$$

We call $\mathcal{M}$ the movement matrix of system (1). To emphasise that there are two types of patches, we also consider a "split" form. To formulate it, first define

$$
\mathbf{P}_{s}=\left(P_{1}, \ldots, P_{S}\right)^{T} \quad \text { and } \quad \mathbf{P}_{t}=\left(P_{S+1}, \ldots, P_{N}\right)^{T} \text {. }
$$

Recall that, by assumption, ordering of patches is with source patches first. If $S=0$ or $S=N$, the corresponding vector $\mathbf{P}_{s}$ or $\mathbf{P}_{t}$, respectively, is vacuous. Then,

$$
\begin{aligned}
& \mathbf{P}_{s}^{\prime}=\mathbf{G}_{s}\left(\mathbf{P}_{s}\right) \mathbf{P}_{s}+\mathcal{M}_{s} \mathbf{P}_{s}+\mathcal{M}_{s t} \mathbf{P}_{t} \\
& \mathbf{P}_{t}^{\prime}=-\mathcal{D}_{t} \mathbf{P}_{t}+\mathcal{M}_{t} \mathbf{P}_{t}+\mathcal{M}_{t s} \mathbf{P}_{s}
\end{aligned}
$$

where

$$
\mathbf{G}_{s}\left(\mathbf{P}_{s}\right)=\operatorname{diag}\left(r_{1}\left(1-\frac{P_{1}}{K_{1}}\right), \ldots, r_{S}\left(1-\frac{P_{S}}{K_{S}}\right)\right)
$$

and

$$
\mathcal{D}_{t}=\operatorname{diag}\left(r_{S+1}, \ldots, r_{N}\right) .
$$

The matrices $\mathcal{M}_{s}, \mathcal{M}_{t}, \mathcal{M}_{s t}$ and $\mathcal{M}_{t s}$ describe movement within and between source and sink patches. They are obtained by writing the movement matrix $\mathcal{M}$ in block form as

$$
\mathcal{M}=\left(\begin{array}{ll}
\mathcal{M}_{s} & \mathcal{M}_{s t} \\
\mathcal{M}_{t s} & \mathcal{M}_{t}
\end{array}\right) .
$$

As a consequence, the matrix $\mathcal{M}_{s}$ is $S \times S, \mathcal{M}_{t}$ is $(N-S) \times(N-S), \mathcal{M}_{s t}$ is $S \times(N-S)$ and $\mathcal{M}_{t s}$ is $(N-S) \times S$.

\section{Mathematical analysis}

In what follows, we work our way towards a proof of the following theorem.

Theorem 1. There exists a unique critical interval $\mathcal{S}_{\text {int }} \subset(0, N) \subset \mathbb{R}$ such that if the number of source patches is $S<\min \left(\mathcal{S}_{\text {int }}\right)$, the population-free equilibrium (PFE) $\left(P_{1}, \ldots, P_{N}\right)=(0, \ldots, 0)$ of System (1) is locally asymptotically stable and if $S>\max \left(\mathcal{S}_{\text {int }}\right)$, the PFE is unstable.

If, additionally, the digraph of patches is strongly connected, then $\mathcal{S}_{\text {int }}$ reduces to a single point, $S^{c}$, and the PFE is globally asymptotically stable in the case that $S<S^{c}$; in the case that $S>S^{c}$, there is a unique componentwise positive equilibrium $\mathbf{P}^{*}$ that is globally asymptotically stable with respect to $\mathbb{R}_{+}^{N} \backslash\{0\}$. 
Note that even in the reducible case, $\mathcal{S}_{\text {int }}$ could be reduced to a single point (we show later in Figure 2 a numerical example where this is the case). Also, values of the number $S$ of source patches are in $\mathbb{N}$, but $\min \left(\mathcal{S}_{\text {int }}\right), \max \left(\mathcal{S}_{\text {int }}\right), S^{c} \in$ $\mathbb{R}$. So, in practice, if $\min \left(\mathcal{S}_{\text {int }}\right), \max \left(\mathcal{S}_{\text {int }}\right)$ or $S^{c} \in \mathbb{R} \backslash \mathbb{N}$, stability properties of the equilibria might not be established for one or several values of $S$.

\subsection{Matrix preliminaries}

Before proceeding further, let us give a few definitions and notations that are used in the remainder of this work.

The vector $\mathbb{1}$ is the vector of all ones, $\mathbb{I}$ is the identity matrix and $\mathbb{J}$ is the matrix of all ones; sizes are indicated only if omission leads to ambiguities. For a vector or matrix $A$, we write $A \geq \mathbf{0}$ to indicate that all entries are nonnegative, $A>\mathbf{0}$ when entries are nonnegative and that there exists at least one nonzero entry; the notation $A \gg \mathbf{0}$ means that $A$ is entry-wise positive. Furthermore, if $A$ and $B$ are vectors or matrices of compatible sizes, we write $A \geq B, A>B$ and $A \gg B$ if $A-B \geq \mathbf{0}, A-B>\mathbf{0}$ and $A-B \gg \mathbf{0}$, respectively.

A matrix $A=\left[a_{i j}\right]$ has the $Z$ sign-pattern or is a $Z$-matrix if $a_{i j} \leq 0$ for all $i \neq j$. A matrix $A$ is essentially nonnegative if $-A$ has the $Z$ sign-pattern. A matrix $A$ is a nonsingular M-matrix, or a matrix of class $K$, if it has the $Z$ signpattern and satisfies any of the equivalent conditions in [9, Theorem 6.2.3], [14, Theorem 5.1] or [24, Theorem 2.5.3]. The matrix is an M-matrix or is of class $K_{0}$ if it has the $Z$ sign-pattern and satisfies any of the equivalent conditions in [14, Theorem 5.2.1]. By [14, Theorem 5.3], $K \subset K_{0}$, with $A \in K_{0}$ also in $K$ if and only if $A$ is nonsingular.

Finally, given a matrix $A \in M_{n}$, the spectrum $\sigma(A)$ is the set of eigenvalues of $A$, the spectral abscissa (or stability modulus) of $A$ is

$$
s(A)=\max \{\Re(\lambda): \lambda \in \sigma(A)\}
$$

and the spectral radius of $A$ is

$$
\rho(A)=\max \{|\lambda|: \lambda \in \sigma(A)\} .
$$

\subsection{Properties of the movement matrix}

The matrix (3) and diagonal perturbations of (3) provide the backbone to much of the analysis. Their main properties are summarised in the following results. Proofs of some parts of these result are given in [5] and the references therein. Very similar results are provided by [36, Corollary 4.3.2], which does not involve M-matrices.

Lemma 2. Consider the movement matrix $\mathcal{M}$ given by (3). Then the following hold true.

1. 0 is an eigenvalue of $\mathcal{M}$ corresponding to the left eigenvector $\mathbb{1}^{T}$.

2. $-\mathcal{M}$ is a singular $M$-matrix. 
3. $0=s(\mathcal{M}) \in \sigma(\mathcal{M})$.

4. If $\mathcal{M}$ is irreducible, then $s(\mathcal{M})$ has multiplicity 1.

Proof. See [5].

We now consider the effect of perturbations of the movement matrix by means of addition of diagonal matrices. In the following statement, $\operatorname{diag}(D)$ refers to the diagonal entries of diagonal matrix $D$.

Proposition 3. Consider the movement matrix $\mathcal{M}$ given by (3). Let $D$ be a diagonal matrix. Then the following hold true.

1. $s(\mathcal{M}+d \mathbb{I})=d$ for all $d \in \mathbb{R}$.

2. $s(\mathcal{M}+D) \in \sigma(\mathcal{M}+D)$ and is associated to an eigenvector $\mathbf{v}>\mathbf{0}$. If additionally, $\mathcal{M}$ is irreducible, then $s(\mathcal{M}+D)$ has multiplicity 1 and is associated to $\mathbf{v} \gg \mathbf{0}$.

3. If $\operatorname{diag}(D) \gg \mathbf{0}$, then $D-\mathcal{M}$ is a nonsingular $M$-matrix and $(D-\mathcal{M})^{-1}>$ 0 .

4. If $\mathcal{M}$ is irreducible and $\operatorname{diag}(D)>\mathbf{0}$, then $D-\mathcal{M}$ is an irreducible nonsingular $M$-matrix and $(D-\mathcal{M})^{-1} \gg \mathbf{0}$.

Proof. 1. From Lemma $2(3), s(\mathcal{M})=0$. Therefore, using a "spectrum shift" [25, Problem 1.2.P8], $s(\mathcal{M}+d \mathbb{I})=d$.

2. These are direct consequences of applying the Perron-Frobenius Theorem to the essentially nonnegative matrix $\mathcal{M}+D$.

3. Define $\underline{d}=\min _{i=1, \ldots, N} d_{i i}$. By the assumption that $\operatorname{diag}(D) \gg \mathbf{0}, \underline{d}>0$. Then $-\mathcal{M} \leq d \mathbb{I}-\mathcal{M} \leq D-\mathcal{M}$. From [14, Theorem 5.2.5], $d \mathbb{I}-\mathcal{M}$ is an M-matrix. Since $s(\mathcal{M})=0$, using a "spectrum shift" [25, Problem 1.2.P8], all eigenvalues of $d \mathbb{I}-\mathcal{M}$ have real parts larger than $\underline{d}$, so $\underline{d} \mathbb{I}-\mathcal{M}$ is a nonsingular M-matrix. In turn, [14, Theorem 5.1.1(4)] implies that $D-\mathcal{M}$ is a nonsingular M-matrix and [14, Theorem 5.1.1(11)] leads to the conclusion.

4. Suppose that $\mathcal{M}$ is irreducible. Let $\bar{d}=\max _{i=1, \ldots, N} d_{i i}>0$. Then $D-\mathcal{M}$ is irreducible and diagonally dominant with all columns $k=1, \ldots, N$ such that $d_{k k}=\bar{d}$ satisfying the strict diagonal dominance requirement. (Other columns with nonzero entries in $D$ also satisfy the requirement.) As a consequence, [39, Theorem 1.11] implies that $D-\mathcal{M}$ is nonsingular and inverse positivity follows from [14, Theorem 5.2.10].

Note that $-\mathcal{M}$ is also the Laplacian matrix of a directed graph. As such, finer estimates of the location of eigenvalues are available; see, e.g., [1]. However, the main concern here is with the spectral abscissa, so this is not needed. 


\subsection{Existence of a threshold $S^{c}$}

In this section, we prove the first part of Theorem 1. We do this in several steps.

In block format, the Jacobian matrix of (4) takes the form

$$
J\left(\mathbf{P}_{s}, \mathbf{P}_{t}\right)=\left(\begin{array}{cc}
\mathbf{G}_{s}^{\prime}\left(\mathbf{P}_{s}\right) \mathbf{P}_{s}+\mathbf{G}_{s}\left(\mathbf{P}_{s}\right)+\mathcal{M}_{s} & \mathcal{M}_{s t} \\
\mathcal{M}_{t s} & -\mathcal{D}_{t}+\mathcal{M}_{t}
\end{array}\right),
$$

where $\mathbf{G}_{s}^{\prime}\left(\mathbf{P}_{s}\right)$ is the term by term derivative of the birth function matrix, i.e.,

$$
\mathbf{G}_{s}^{\prime}\left(\mathbf{P}_{s}\right)=\operatorname{diag}\left(-\frac{r_{1}}{K_{1}}, \ldots,-\frac{r_{S}}{K_{s}}\right) .
$$

So, at the PFE, denoting $J_{\mathrm{PFE}}:=J(0,0)$ and $\mathcal{D}_{s}=\operatorname{diag}\left(r_{1}, \ldots, r_{S}\right)$,

$$
\begin{aligned}
J_{\mathrm{PFE}} & =\left(\begin{array}{cc}
\mathbf{G}_{s}(\mathbf{0})+\mathcal{M}_{s} & \mathcal{M}_{s t} \\
\mathcal{M}_{t s} & \mathcal{M}_{t}-\mathcal{D}_{t}
\end{array}\right) \\
& =\left(\begin{array}{cc}
\operatorname{diag}\left(r_{1}, \ldots, r_{S}\right)+\mathcal{M}_{s} & \mathcal{M}_{s t} \\
\mathcal{M}_{t s} & \mathcal{M}_{t}-\mathcal{D}_{t}
\end{array}\right) \\
& =\left(\operatorname{diag}\left(r_{1}, \ldots, r_{S}\right) \oplus \operatorname{diag}\left(-r_{S+1}, \ldots,-r_{N}\right)\right)+\mathcal{M}
\end{aligned}
$$

Thus,

$$
J_{\mathrm{PFE}}^{S}=\mathcal{M}+\left(\mathcal{D}_{s} \oplus-\mathcal{D}_{t}\right)
$$

where, in the matrix $\mathcal{D}_{s} \oplus-\mathcal{D}_{t}=\operatorname{diag}\left(r_{1}, \ldots, r_{S},-r_{S+1}, \ldots,-r_{N}\right)$, there are $S$ positive entries and $N-S$ negative entries. To make the balance of positive and negative terms apparent, we use the notation $J_{\mathrm{PFE}}^{S}$.

In order to conduct the analysis, we need a way to continuously change values in the matrix. For $S=0, \ldots, N-1$, denote

$$
J_{\mathrm{PFE}}^{S, \varepsilon}=\mathcal{M}+\operatorname{diag}\left(r_{1}, \ldots, r_{S}, \varepsilon,-r_{S+2}, \ldots,-r_{N}\right),
$$

where $\varepsilon \in\left[-r_{S+1}, r_{S+1}\right]$ is in the $(S+1)^{\text {th }}$ position in the diagonal matrix. We then have

$$
J_{\mathrm{PFE}}^{S,-r_{S+1}}=J_{\mathrm{PFE}}^{S} \quad \text { and } \quad J_{\mathrm{PFE}}^{S, r_{S+1}}=J_{\mathrm{PFE}}^{S+1} .
$$

It is also useful to be able to refer to $S$ as a continuous variable, even if this is an abuse of notation when referring to the number of source patches. In this case, for $\zeta \in[0, N]$, we consider

$$
J_{\mathrm{PFE}}^{\zeta}=J_{\mathrm{PFE}}^{\xi, \varepsilon}, \quad \text { with } \quad \xi=\lfloor\zeta\rfloor, \quad \varepsilon=2(\zeta-\lfloor\zeta\rfloor) r_{i}-r_{i},
$$

where $i=\lfloor\zeta\rfloor+1$ if $\zeta<N$ and $i=N$ when $\zeta=N$. In the sequel, we use the notation $J_{\mathrm{PFE}}^{S}$ to indicate that $S \in[0, N]$ and $J_{\mathrm{PFE}}^{\xi, \varepsilon}$ to specify an integer value $\xi$ with offset $\varepsilon$.

We can now proceed with the proof of the first part of Theorem 1. First, we prove the following.

Lemma 4. Let $\underline{r}=\min _{i=1 \ldots N}\left\{r_{i}\right\}$. Then, when $S=0, s\left(J_{P F E}^{0}\right) \leq-\underline{r}<0$, whereas $s\left(J_{P F E}^{N}\right) \geq \underline{r}>0$ when $S=N$. 
Proof. If $S=0$, then

$$
J_{\mathrm{PFE}}^{0}=\mathcal{M}+\operatorname{diag}\left(-r_{1}, \ldots,-r_{N}\right) .
$$

From Proposition 2(3), $s(\mathcal{M})=0$. Note that this follows from using the Gershgorin Theorem, where for $\mathcal{M}$, all Gershgorin disks are left of the imaginary axis and tangent to origin of the complex plane. Then the centres of the Gershorin disks of $\mathcal{M}+\operatorname{diag}\left(-r_{1}, \ldots,-r_{N}\right)$ are shifted left by $r_{1}, \ldots, r_{N}$ while the radii remain the same. As a consequence, the closest disk(s) to the origin of the complex plane have centre $(\mathrm{s})-\underline{r}$ and thus $s\left(\mathcal{M}+\operatorname{diag}\left(-r_{1}, \ldots,-r_{N}\right)\right) \leq-\underline{r}<0$.

If $S=N$, then

$$
J_{\mathrm{PFE}}^{N}=\mathcal{M}+\operatorname{diag}\left(r_{1}, \ldots, r_{N}\right) .
$$

For $i=1, \ldots, N$, define $e_{i}=r_{i}-\underline{r} \geq 0$, then

$$
J_{\mathrm{PFE}}^{N}=\mathcal{M}+\underline{r} \mathbb{I}+\operatorname{diag}\left(e_{1}, \ldots, e_{N}\right),
$$

where, by Proposition 3(1), $s(\mathcal{M}+\underline{r} \mathbb{I})=\underline{r}>0$.

First, assume $\mathcal{M}$ is irreducible. Then $J_{\mathrm{PFE}}^{N}$ is an irreducible essentially nonnegative matrix and, since $J_{\mathrm{PFE}}^{N} \geq \mathcal{M}+\underline{r} \mathbb{I}$, it follows from [36, Corollary 4.3.2(3)] that $s\left(J_{\mathrm{PFE}}^{N}\right) \geq s(\mathcal{M}+\underline{r} \mathbb{I})=\underline{r}$, with the inequalities being strict if there exists at least one $e_{i}>0$.

Now assume that $\mathcal{M}$ is reducible. Then there exists a permutation matrix $P$ such that $P^{T} \mathcal{M P}$ is block upper triangular with irreducible blocks on the diagonal. Call $C$ the number of such blocks, i.e., the number of strong components in the digraph of patches. For $i=1, \ldots, C$, denote $n(i)$ the number of patches in strong component $i$ and $k(1), \ldots, k(n(i))$ their indices. By abuse of notation, denote $\mathcal{M}_{i i}$ the corresponding diagonal block in the reduced form of $\mathcal{M}$.

Applying the permutation matrix $P$ to $J_{\mathrm{PFE}}^{N}$ gives a block upper triangular matrix $P^{T} J_{\mathrm{PFE}}^{N} P$ with, for $i=1, \ldots, C$, the $n(i) \times n(i)$ diagonal blocks $\mathcal{M}_{i i}+E_{i}$ being irreducible and with

$$
E_{i}=\underline{r} \mathbb{I}+\operatorname{diag}\left(e_{k(1)}, \ldots, e_{k(n(i))}\right) .
$$

Fix $i=1, \ldots, C$ and let $\mathbf{v}$ be a positive right eigenvector of $\mathcal{M}_{i i}+E_{i}$ corresponding to the spectral abscissa $s_{1}$ and $\mathbf{w}$ be a positive left eigenvector of $\mathcal{M}_{i i}+\underline{r} \mathbb{I}$ corresponding to the spectral abscissa $s_{2}$. Then

$$
\begin{aligned}
s_{1} \mathbf{w}^{T} \mathbf{v} & =\mathbf{w}^{T}\left(\mathcal{M}_{i i}+\underline{r} \mathbb{I}+\operatorname{diag}\left(e_{k(1)}, \ldots, e_{k(n(i))}\right) \mathbf{v}\right. \\
& =\mathbf{w}^{T}\left(\mathcal{M}_{i i}+\underline{r} \mathbb{I}\right) \mathbf{v}+\mathbf{w}^{T} \operatorname{diag}\left(e_{k(1)}, \ldots, e_{k(n(i))}\right) \mathbf{v} \\
& =s_{2} \mathbf{w}^{T} \mathbf{v}+\mathbf{w}^{T} \operatorname{diag}\left(e_{k(1)}, \ldots, e_{k(n(i))}\right) \mathbf{v} \\
& \geq s_{2} \mathbf{w}^{T} \mathbf{v},
\end{aligned}
$$

the inequality being strict if at least one of the $e_{k(j)}, j=1, \ldots, n(i)$, is positive. Hence $s_{1} \geq s_{2}$, i.e., $s\left(\mathcal{M}_{i i}+E_{i}\right) \geq s\left(\mathcal{M}_{i i}+\underline{r} \mathbb{I}\right)$. This is true for all diagonal blocks. Now, since $P^{T} J_{\mathrm{PFE}}^{N} P$ is block upper triangular,

$$
s\left(J_{\mathrm{PFE}}^{N}\right)=s\left(P^{T} J_{\mathrm{PFE}}^{N} P\right)=\max \left\{s\left(\mathcal{M}_{11}+E_{1}\right), \ldots, s\left(\mathcal{M}_{C C}+E_{C}\right)\right\} .
$$


As $P^{T}(\mathcal{M}+\underline{r} \mathbb{I}) P$ is also block upper triangular,

$$
\underline{r}=s(\mathcal{M}+\underline{r} \mathbb{I})=\max \left\{s\left(\mathcal{M}_{11}+\underline{r} \mathbb{I}\right), \ldots, s\left(\mathcal{M}_{11}+\underline{r} \mathbb{I}\right)\right\} .
$$

As a consequence, $s\left(J_{\mathrm{PFE}}^{N}\right) \geq \underline{r}>0$.

Thus, $S^{c}$ necessarily lies in the open interval $(0, N)$. The following lemma is of interest and the method of proof of the second assertion is used again later.

Lemma 5. 1. For all $S \in(0, N) \subset \mathbb{R}$,

$$
J_{P F E}^{0}<J_{P F E}^{S}<J_{P F E}^{N} .
$$

2. $J_{P F E}^{S}$ is an increasing function of $S$, in the sense that

$$
\forall S_{1}, S_{2} \in[0, N] \subset \mathbb{R} \text { such that } S_{1}<S_{2}, \quad J_{P F E}^{S_{1}}<J_{P F E}^{S_{2}} .
$$

Proof. 1. Let $S \in(0, N)$ be fixed. Using (9), this gives a pair $(\xi, \varepsilon) \in$ $\{0, \ldots, N\} \times\left[-r_{i}, r_{i}\right]$, for $i=1 \ldots N$, such that $J_{\mathrm{PFE}}^{S}=J_{\mathrm{PFE}}^{\xi, \varepsilon}$. We have

$$
\begin{aligned}
J_{\mathrm{PFE}}^{\xi, \varepsilon}-J_{\mathrm{PFE}}^{0}= & \mathcal{M}+\operatorname{diag}\left(r_{1}, \ldots, r_{\xi}, \varepsilon,-r_{\xi+2}, \ldots,-r_{N}\right) \\
& -\mathcal{M}-\operatorname{diag}\left(-r_{1}, \ldots,-r_{N}\right) \\
= & \operatorname{diag}\left(2 r_{1}, \ldots, 2 r_{\xi}, \varepsilon+r_{\xi+1}, 0, \ldots, 0\right) \\
> & \mathbf{0}
\end{aligned}
$$

since $\varepsilon \in\left[-r_{\xi+1}, r_{\xi+1}\right]$. Computing $J_{\mathrm{PFE}}^{N}-J_{\mathrm{PFE}}^{\xi, \varepsilon}$ at the other endpoint works similarly, giving (10).

2. Use (9) again to obtain two pairs $\left(\xi_{1}, \varepsilon_{1}\right)$ and $\left(\xi_{2}, \varepsilon_{2}\right)$, where, by the assumption $S_{1}<S_{2}, \xi_{1} \leq \xi_{2}$. First, assume that $\xi_{1}<\xi_{2}$. Then

$$
\begin{aligned}
J_{\mathrm{PFE}}^{\xi_{2}, \varepsilon_{2}}-J_{\mathrm{PFE}}^{\xi_{1}, \varepsilon_{1}}= & \operatorname{diag}\left(r_{1}, \ldots, r_{\xi_{2}}, \varepsilon_{2},-r_{\xi_{2}+2}, \ldots,-r_{N}\right) \\
& -\operatorname{diag}\left(r_{1}, \ldots, r_{\xi_{1}}, \varepsilon_{1},-r_{\xi_{1}+2}, \ldots,-r_{N}\right) \\
= & \operatorname{diag}\left(0, \ldots, 0, r_{\xi_{1}+1}-\varepsilon_{1}, 2 r_{\xi_{1}+2}, \ldots, 2 r_{\xi_{2}}, \varepsilon_{2}+r_{\xi_{2}+1}, 0, \ldots, 0\right) \\
> & \mathbf{0}
\end{aligned}
$$

since $\varepsilon_{1} \in\left[-r_{\xi_{1}+1}, r_{\xi_{1}+1}\right]$, and $\varepsilon_{2} \in\left[-r_{\xi_{2}+1}, r_{\xi_{2}+1}\right]$. Now assume $\xi_{1}=\xi_{2}$. Then, since $S_{1}<S_{2}$, we find that $\varepsilon_{1}<\varepsilon_{2}$ and the diagonal matrix in the subtraction $J_{\mathrm{PFE}}^{\xi_{2}, \varepsilon_{2}}-J_{\mathrm{PFE}}^{\xi_{2}, \varepsilon_{1}}$ takes the form $\operatorname{diag}\left(0, \ldots, 0, \varepsilon_{2}-\varepsilon_{1}, 0, \ldots, 0\right)>\mathbf{0}$. So (11) holds.

Proposition 6. If $\mathcal{M}$ is irreducible, the spectral abscissa $s\left(J_{P F E}^{S}\right)$ is an increasing function of $S \in[0, N] \subset \mathbb{R}$. If $\mathcal{M}$ is reducible, the spectral abscissa $s\left(J_{P F E}^{S}\right)$ is a nondecreasing function of $S \in[0, N] \subset \mathbb{R}$.

Proof. First, assume $\mathcal{M}$ is irreducible. Then, by Lemma 5 and the fact that $\mathcal{M}$ is irreducible (and thus so is $J_{\mathrm{PFE}}^{S}$ ), [36, Corollary 4.3.2(3)] gives the result.

Now, assume that $\mathcal{M}$ is reducible. Then there exists a permutation matrix $P$ such that $P^{T} \mathcal{M} P$ is block upper triangular. Consider $S \in[0, N] \subset \mathbb{R}$ and 
use (9) to obtain a corresponding pair $(\xi, \varepsilon) \in\{0, \ldots, N\} \times\left[-r_{\xi}, r_{\xi}\right]$. Apply the same permutation to $J_{\mathrm{PFE}}^{\xi, \varepsilon}$, giving

$$
P^{T} J_{\mathrm{PFE}}^{\xi, \varepsilon} P=\left(\begin{array}{cccc}
\mathcal{M}_{11}+E_{1} & \mathcal{M}_{12} & \cdots & \mathcal{M}_{1 N} \\
0 & \mathcal{M}_{22}+E_{2} & & \\
& & \ddots & \\
0 & \ldots & 0 & \mathcal{M}_{C C}+E_{C}
\end{array}\right),
$$

where $C$ is the number of strong components in the digraph of patches and

$$
E_{1} \oplus \cdots \oplus E_{C}=P^{T} \operatorname{diag}\left(r_{1}, \ldots, r_{\xi}, \varepsilon,-r_{\xi+2}, \ldots,-r_{N}\right) P
$$

with the matrix on the right hand side having $\varepsilon$ as $(\xi+1)^{\text {th }}$ diagonal entry. As in the proof of Lemma 4, we have denoted $\mathcal{M}_{i i}$ the diagonal blocks in the reduced form of $\mathcal{M}$.

For $j=1, \ldots, C$, each of the matrices $\mathcal{M}_{j j}$ is irreducible; $C-1$ of the matrices $E_{j}$ are diagonal with entries $-r_{i}$ and $r_{i}$ on the diagonal (with some having only $-r_{i}$, some having only $r_{i}$ and some having both types of entries). The remaining $E_{j}$ matrix is diagonal, with potentially $-r_{i}$ and $r_{i}$ as the others, but also $\varepsilon$. Let us call $\eta \in\{1, \ldots, C\}$ the index of the strong component containing the matrix with $\varepsilon$.

As a consequence, for all $j=1, \ldots, C, \mathcal{M}_{j j}+E_{j}$ are irreducible essentially nonnegative matrices, with only matrix $\mathcal{M}_{\eta \eta}+E_{\eta}$ having an $\varepsilon$ added to one of its diagonal entries.

As $P^{T} J_{\mathrm{PFE}}^{\xi, \varepsilon} P$ is block upper triangular, we have

$$
s\left(P^{T} J_{\mathrm{PFE}}^{\xi, \varepsilon} P\right)=\max \left\{s\left(\mathcal{M}_{11}+E_{1}\right), \ldots, s\left(\mathcal{M}_{C C}+E_{C}\right)\right\} .
$$

Except for $\mathcal{M}_{\eta \eta}+E_{\eta}$, all matrices $\mathcal{M}_{i i}+E_{i}$ have fixed spectral abscissa. Concerning matrix $\mathcal{M}_{\eta \eta}+E_{\eta}$, it is clear that the reasoning in the proof of Lemma 5(2) carries through and thus,

$$
\forall \varepsilon_{1}, \varepsilon_{2} \in\left[-r_{\xi+1}, r_{\xi+1}\right], \varepsilon_{1}<\varepsilon_{2} \Longrightarrow J_{\mathrm{PFE}}^{\xi, \varepsilon_{1}}<J_{\mathrm{PFE}}^{\xi, \varepsilon_{2}} .
$$

Hence $s\left(J_{\mathrm{PFE}}^{\xi, \varepsilon}\right)$ is the maximum of a set of $C$ functions, $C-1$ of which are constant in $\varepsilon$ and one of which is increasing in $\varepsilon$. It now follows that $s\left(J_{\mathrm{PFE}}^{S}\right)$ is a nondecreasing function of $S$, as desired.

We are now in a position to prove the first part of Theorem 1.

Proof (first part of Theorem 1). As $J_{\mathrm{PFE}}^{S}$ is an essentially nonnegative matrix, its spectral abscissa $s\left(J_{\mathrm{PFE}}^{S}\right)$ is an eigenvalue. Eigenvalues of $J_{\mathrm{PFE}}^{S}$ depend continuously on $S$ [25, Theorem 2.4.9.2]. By Lemma 4, $s\left(J_{\mathrm{PFE}}^{0}\right)<0$ and $s\left(J_{\mathrm{PFE}}^{N}\right)>0$, so by the Intermediate Value Theorem, there exists at least one point $S^{c} \in(0, N)$ such that $s\left(J_{\mathrm{PFE}}^{S^{c}}\right)=0$. In the case where $\mathcal{M}$ is irreducible, $s\left(J_{\mathrm{PFE}}^{S}\right)$ is increasing by Proposition 6 and as a consequence, $S^{c}$ is unique. In the case where $\mathcal{M}$ is reducible, $s\left(J_{\mathrm{PFE}}^{S}\right)$ is nondecreasing, therefore there exists 
an interval $\mathcal{S}_{i n t}$, possibly reduced to a single point, such that $s\left(J_{\mathrm{PFE}}^{S}\right)=0$ for all $S \in \mathcal{S}_{\text {int }}$.

The usual criteria for local asymptotic stability and instability of equilibria then imply the first part of Theorem 1 for $S<S^{c}$ and $S>S^{c}$ (irreducible case) or $S<\min \left(\mathcal{S}_{\text {int }}\right)$ and $S>\max \left(\mathcal{S}_{\text {int }}\right)$ (reducible case).

Example 1. Here we consider a small order example that illustrates how, when the movement matrix is somewhat heterogeneous, the ordering of its rows and columns can affect the value of $S^{c}$. Specifically let $\mathcal{M}$ be given by

$$
\mathcal{M}=\left(\begin{array}{ccc}
-4 & 0 & 1 \\
0 & -4 & 1 \\
4 & 4 & -2
\end{array}\right)
$$

and for convenience suppose that $r=1$. It is straightforward to verify that the spectral abscissa of $\mathcal{M}+\operatorname{diag}(1,1,-1)$ is negative (since the characteristic polynomial has all coefficients positive), and since the spectral abscissa of $\mathcal{M}+$ $\operatorname{diag}(1,1,1)$ is positive, we deduce that $S^{c} \in(2,3)$.

Next let $\widehat{\mathcal{M}}$ be given by

$$
\widehat{\mathcal{M}}=\left(\begin{array}{ccc}
-2 & 4 & 4 \\
1 & -4 & 0 \\
1 & 0 & -4
\end{array}\right),
$$

and observe that $\widehat{\mathcal{M}}$ is permutationally similar to $\mathcal{M}$. A computation reveals that the spectral abscissa of $\widehat{\mathcal{M}}+\operatorname{diag}(1,-1,-1)$ is positive (since the constant term of the characteristic polynomial is negative), so that $S^{c} \in(0,1)$ in this case. Thus we see that while the two movement matrices $\mathcal{M}$ and $\widehat{\mathcal{M}}$ carry the same information, our ordering convention that puts all source patches first is influential on the corresponding threshold number $S^{c}$.

Example 2. Consider the reducible movement matrix

$$
\mathcal{M}=\left(\begin{array}{ccccc}
-2 & 1 & 1 & 1 & 1 \\
0 & -4 & 1 & 0 & 0 \\
0 & 1 & -4 & 0 & 0 \\
1 & 1 & 1 & -2 & 1 \\
1 & 1 & 1 & 1 & -2
\end{array}\right)
$$

and suppose $r_{i}=1$ for $i=1, \ldots, 5$. Then we find $s\left(J_{\mathrm{PFE}}^{2}\right)=s\left(J_{\mathrm{PFE}}^{3}\right)=$ $s\left(J_{\mathrm{PFE}}^{4}\right)=0$, so $\mathcal{S}_{\text {int }} \supseteq[2,4]$. Thus, clearly, in the reducible case, $\mathcal{S}_{\text {int }}$ can be an interval.

\subsection{Boundedness of solutions}

Before we proceed with the case of an irreducible movement matrix, we must establish boundedness of solutions. First, remark that it is clear that solutions to 
(1) exist for all $t \geq 0$ and remain nonnegative for nonnegative initial conditions. Thus, $\mathbb{R}_{+}^{N}$ is invariant under the flow of (1).

Recall the Jacobian matrix at an arbitrary point given by (6). Clearly, this matrix has nonnegative off-diagonal entries. As a consequence, system (1) is cooperative.

Proposition 7. Solutions to (1) are bounded.

Proof. Recall that a logistic equation can also be written in the form

$$
P_{i}^{\prime}=b_{i} P_{i}-d_{i} P_{i}-c_{i} P_{i}^{2},
$$

where $b_{i}, d_{i}$ and $c_{i}$ are, respectively, the birth, death and competition induced death rates. Setting $r_{i}=b_{i}-d_{i}$ and $K_{i}=\left(b_{i}-d_{i}\right) / c_{i}$ then gives the logistic equation used earlier. We have

$$
\begin{aligned}
\left(\sum_{i=1}^{N} P_{i}\right)^{\prime} & =\sum_{i=1}^{S}\left(b_{i} P_{i}-d_{i} P_{i}-c_{i} P_{i}^{2}\right)-\sum_{i=S+1}^{N} r_{i} P_{i} \\
& \leq \sum_{i=1}^{S}\left(\bar{b} P_{i}-\underline{d} P_{i}-\underline{c} P_{i}^{2}\right),
\end{aligned}
$$

where $\bar{b}=\max _{i=1, \ldots, S}\left\{b_{i}\right\}, \underline{d}=\min _{i=1, \ldots, S}\left\{d_{i}\right\}$ and $\underline{c}=\min _{i=1, \ldots, S}\left\{c_{i}\right\}$. Because, by assumption, $K_{i}>0$ for all $i=1, \ldots, S$, it is reasonable to assume that the same is true of $c_{i}$ (otherwise the system would not be limited by competition). Hence $\underline{c}>0$. As a consequence, defining $\tilde{r}=\bar{b}-\underline{d}$ and $\tilde{K}=(\bar{b}-\underline{d}) / \underline{c}$, we have

$$
\left(\sum_{i=1}^{N} P_{i}\right)^{\prime} \leq \sum_{i=1}^{S} \tilde{r} P_{i}\left(1-\frac{P_{i}}{\tilde{K}}\right) .
$$

Since (1) is cooperative in the positive orthant and parameters are positive, it follows that for $t \geq 0$,

$$
\sum_{i=1}^{N} P_{i}(t) \leq \sum_{i=1}^{S} \frac{\tilde{K} P_{i}(0)}{P_{i}(0)+\left(\tilde{K}-P_{i}(0)\right) e^{-\tilde{r} t}} .
$$

Thus, as $t \rightarrow \infty$,

$$
\sum_{i=1}^{N} P_{i}(t) \leq S \tilde{K}
$$

As a consequence, since the positive orthant is invariant under the flow of (1), solutions to (1) are bounded.

\subsection{Case of an irreducible movement matrix}

Now assume that the movement matrix is irreducible, or, equivalently, that the digraph of patches is strongly connected. The global stability of the PFE when $S<S^{c}$ and existence and global stability of a (strongly) positive equilibrium when $S>S^{c}$ then follow from [22, Theorem 6.1]. 
Proof (second part of Theorem 1). In the case of (1), the condition in [22, Theorem 6.1] that the Jacobian matrix $J^{S}$ be strictly antimonotone is not satisfied, since $\mathbf{P}_{1}<\mathbf{P}_{2}$ does not imply that $J_{\mathbf{P}_{1}}^{S}>J_{\mathbf{P}_{2}}^{S}$ in the case of vectors $\mathbf{P}_{1}, \mathbf{P}_{2}$ with first $S$ entries equal to zero and other entries positive, instead leading to $J_{\mathbf{P}_{1}}^{S}=J_{\mathbf{P}_{2}}^{S}$.

However, it is possible to use the method of proof of [22, Theorem 6.1] conclusively. All other assumptions of that result are satisfied: in the case that $\mathcal{M}$ is irreducible, (1) is strongly monotone (by [22, Theorem 1.7]); the origin is an equilibrium; all solutions in $\mathbb{R}_{+}^{N}$ are bounded (by Proposition 7 ). Therefore, as in the proof of [22, Theorem 6.1], there exists an equilibrium $\mathbf{P}^{*} \gg \mathbf{0}$.

Strict antimonotonicity is then used to show uniqueness of $\mathbf{P}^{*}$. Indeed, antimonotonicity of the vector field $F$ of the system under consideration implies that, if $\mathbf{P}^{*}$ is a nonzero equilibrium of $F$, then, letting $\mathbf{z}$ be in the open line segment having endpoints $\mathbf{0}$ and $\mathbf{P}^{*}$, there holds that $F(\mathbf{z})>\mathbf{0}$, which is then used to conclude. Let us show that $F(\mathbf{z})>\mathbf{0}$ is indeed the case for (1). As in the proof of $\left[22\right.$, Theorem 6.1], define, for each $i=1, \ldots, N$, the map $g_{i}:[0,1] \rightarrow \mathbb{R}$, $g_{i}(s)=F_{i}\left(s \mathbf{P}^{*}\right)$. Then $g_{i}(0)=g_{i}(1)=0$. Hence, for $i=S+1, \ldots, N$,

$$
g_{i}(s)=-r_{i} s P_{i}^{*}+\sum_{j=1}^{N} m_{i j} s P_{j}^{*}=\left(r_{i} P_{i}^{*}+\sum_{j=1}^{N} m_{i j} P_{j}^{*}\right) s=0 .
$$

However, for $i=1, \ldots, S$,

$$
g_{i}(s)=r_{i}\left(1-\frac{s P_{i}^{*}}{K_{i}}\right) s P_{i}^{*}+\sum_{j=1}^{N} m_{i j} s P_{j}^{*} .
$$

Clearly,

$$
g_{i}^{\prime \prime}(s)=-\frac{2 r_{i} P_{i}^{* 2}}{K}<0, \quad i=1, \ldots, S .
$$

As a consequence, for $i=1, \ldots, S, g_{i}(s)>0$ for $s \in(0,1)$. Thus, when $S>0$, $F(\mathbf{z})>\mathbf{0}$ for all $\mathbf{z}$ in the open interval from $\mathbf{0}$ to $\mathbf{P}^{*}$. The remainder of the proof of [22, Theorem 6.1] holds and as a consequence, so do the conclusions of [22, Theorem 6.1]. Thus, all solutions of (1) limit either to zero or to a unique $\mathbf{P}^{*} \gg \mathbf{0}$. From the first part of Theorem 1, this implies the result in terms of $S^{c}$ when $S>0$.

Finally, the case $S=0$ is simple. Indeed, consider the evolution of the total population in this case. We have

$$
\left(\sum_{i=1}^{N} P_{i}\right)^{\prime}=-\sum_{i=1}^{N} r_{i} P_{i}<0
$$

since at least one of the $P_{i}(0)>0$. As a consequence, the total population in the system tends to zero and by the invariance of $\mathbb{R}_{+}^{N}, \lim _{t \rightarrow \infty} P_{i}(t)=0$ for all $i=1, \ldots, N$. This concludes the proof of Theorem 1 . 
In the irreducible case, we can also express the threshold using a basic reproduction number. We have the following.

Proposition 8. Suppose the movement matrix $\mathcal{M}$ is irreducible. Define the basic reproduction number

$$
\mathcal{R}_{0}=\rho\left(\left(\mathcal{M}_{s}+\mathcal{M}_{s t}\left(\mathcal{D}_{t}-\mathcal{M}_{t}\right)^{-1} \mathcal{M}_{t s}\right)^{-1} \mathcal{D}_{s}\right),
$$

where $\mathcal{M}_{s}, \mathcal{M}_{t}, \mathcal{M}_{s t}, \mathcal{M}_{t s}$ are defined as in (5), $\mathcal{D}_{s}$ and $\mathcal{D}_{t}$ are defined as in Section 3.3. Then

$$
s\left(J_{P F E}^{S}\right)<0 \Longleftrightarrow \mathcal{R}_{0}<1 \text { and } s\left(J_{P F E}^{S}\right)>0 \Longleftrightarrow \mathcal{R}_{0}>1 .
$$

Proof. Write (7) as

$$
J_{\mathrm{PFE}}^{S}=\mathcal{M}+\tilde{\mathcal{D}}_{s}-\tilde{D}_{t},
$$

where $\tilde{\mathcal{D}}_{s}=\mathcal{D}_{s} \oplus \mathbf{0}_{N-S \times N-S}$ and $\tilde{\mathcal{D}}_{t}=\mathbf{0}_{S \times S} \oplus \mathcal{D}_{t}$. Let $-\alpha$ be the spectral abscissa of $\mathcal{M}+\tilde{\mathcal{D}}_{s}-\tilde{\mathcal{D}}_{t}$. From Proposition 3(2), there is a vector $\mathbf{v} \gg \mathbf{0}$ such that

$$
\left(\mathcal{M}+\tilde{\mathcal{D}}_{s}-\tilde{\mathcal{D}}_{t}\right) \mathbf{v}=-\alpha \mathbf{v}
$$

In other words,

$$
\alpha \mathbf{v}=\left(\tilde{\mathcal{D}}_{t}-\mathcal{M}\right) \mathbf{v}-\tilde{\mathcal{D}}_{s} \mathbf{v}
$$

By the assumption of irreducibility of $\mathcal{M}$, it follows from Proposition 3(4) that $\tilde{\mathcal{D}}_{t}-\mathcal{M}$ is an irreducible nonsingular M-matrix and $\left(\tilde{\mathcal{D}}_{t}-\mathcal{M}\right)^{-1} \gg \mathbf{0}$. Then

$$
\alpha\left(\tilde{\mathcal{D}}_{t}-\mathcal{M}\right)^{-1} \mathbf{v}=\mathbf{v}-\left(\tilde{\mathcal{D}}_{t}-\mathcal{M}\right)^{-1} \tilde{\mathcal{D}}_{s} \mathbf{v}
$$

with the matrix $\left(\tilde{\mathcal{D}}_{t}-\mathcal{M}\right)^{-1} \tilde{\mathcal{D}}_{s}>\mathbf{0}$. As a consequence, from the PerronFrobenius Theorem, the spectral radius of $\left(\tilde{\mathcal{D}}_{t}-\mathcal{M}\right)^{-1} \tilde{\mathcal{D}}_{s}$ is an eigenvalue and is associated to a nonnegative eigenvector. Let $\mathbf{u}$ be such an eigenvector, normalised so that $\mathbf{u}^{T} \mathbf{v}=1$. Then

$$
\alpha \mathbf{u}^{T}\left(\tilde{\mathcal{D}}_{t}-\mathcal{M}\right)^{-1} \mathbf{v}=\mathbf{u}^{T} \mathbf{v}\left(1-\rho\left\{\left(\tilde{\mathcal{D}}_{t}-\mathcal{M}\right)^{-1} \tilde{\mathcal{D}}_{s}\right\}\right) .
$$

Thus

$$
\alpha>0 \Longleftrightarrow \rho\left\{\left(\tilde{\mathcal{D}}_{t}-\mathcal{M}\right)^{-1} \tilde{\mathcal{D}}_{s}\right\}<1
$$

and

$$
\alpha<0 \Longleftrightarrow \rho\left\{\left(\tilde{\mathcal{D}}_{t}-\mathcal{M}\right)^{-1} \tilde{\mathcal{D}}_{s}\right\}>1
$$

From the structure of $\tilde{\mathcal{D}}_{s}$, the spectral radius of $\left(\tilde{\mathcal{D}}_{t}-\mathcal{M}\right)^{-1} \tilde{\mathcal{D}}_{s}$ is the spectral radius of

$$
\left(\tilde{\mathcal{D}}_{t}-\mathcal{M}\right)_{[11]}^{-1} \mathcal{D}_{s}
$$


where $\left(\tilde{\mathcal{D}}_{t}-\mathcal{M}\right)_{[11]}^{-1}$ is the $(1,1)$ block in $\left(\tilde{\mathcal{D}}_{t}-\mathcal{M}\right)^{-1}$. Writing $\mathcal{M}$ as $(5)$, we have by the formula for the inverse of a $2 \times 2$ block matrix that

$$
\left(\tilde{\mathcal{D}}_{t}-\mathcal{M}\right)_{[11]}^{-1}=\left(-\mathcal{M}_{s}-\mathcal{M}_{s t}\left(\mathcal{D}_{t}-\mathcal{M}_{t}\right)^{-1} \mathcal{M}_{t s}\right)^{-1}
$$

Clearly,

$$
\begin{aligned}
& \rho\left(\left(-\mathcal{M}_{s}-\mathcal{M}_{s t}\left(\mathcal{D}_{t}-\mathcal{M}_{t}\right)^{-1} \mathcal{M}_{t s}\right)^{-1} \mathcal{D}_{s}\right) \\
& \quad=\rho\left(\left(\mathcal{M}_{s}+\mathcal{M}_{s t}\left(\mathcal{D}_{t}-\mathcal{M}_{t}\right)^{-1} \mathcal{M}_{t s}\right)^{-1} \mathcal{D}_{s}\right),
\end{aligned}
$$

giving the result.

We note in passing that the matrix $\left(\mathcal{M}_{s}+\mathcal{M}_{s t}\left(\mathcal{D}_{t}-\mathcal{M}_{t}\right)^{-1} \mathcal{M}_{t s}\right)^{-1} \mathcal{D}_{s}$ of (12) has all negative entries.

Similarly to what happens with metapopulation models in mathematical epidemiology [5], we have an expression for $\mathcal{R}_{0}$ that cannot be evaluated explicitly but is easy to obtain numerically once parameters are fixed.

\subsection{Case of irreducible homogeneous movement}

Suppose that the movement graph is complete and migration rates all equal $m$. Furthermore, assume that growth rates in source patches all equal $r_{s}$ and death rates are equal to $r_{t}$ in all sinks. Then the threshold $S^{c}$ can be computed explicitly.

Proposition 9. Let the movement digraph be complete and movement rates be such that $m_{i j}=m$ for all $i, j=1, \ldots, N, i \neq j$. Assume that $S \in\{1, \ldots, N-1\}$, that for $i=1, \ldots, S, r_{i}=r_{s}$ and that for $i=S+1, \ldots, N, r_{i}=r_{t}$. Then

$$
S^{c}=\frac{m N r_{t}-r_{s} r_{t}}{m\left(r_{s}+r_{t}\right)}
$$

The proof uses the principle of equitable partitions; see [15, Section 9.3]. An equitable partition splits a graph $\mathcal{G}$ into cells $\mathcal{C}_{i}, i=1, \ldots, \ell$, such that, for a given vertex $u$ in cell $\mathcal{C}_{i}$, the number of neighbours in cell $\mathcal{C}_{j}$ is a constant $b_{i j}$ and does not depend on $u$.

Proof. The Jacobian matrix at the PFE can be written as

$$
J_{\mathrm{PFE}}^{S}=\left(\begin{array}{cc}
m \mathbb{J}-N m \mathbb{I}+r_{s} \mathbb{I} & m \mathbb{J} \\
m \mathbb{J} & m \mathbb{J}-N m \mathbb{I}-r_{t} \mathbb{I}
\end{array}\right),
$$

where, in each block, matrices $\mathbb{I}$ and $\mathbb{J}$ have dimensions making them compatible with the operations.

Consider (15) as the adjacency matrix of a digraph $\mathcal{G}$. Suppose that, through a partition $\pi$, we split $\mathcal{G}$ into two cells, $\left\{S_{i}\right\}_{i=1, \ldots, S}$ (sources) and $\left\{T_{i}\right\}_{i=S+1, \ldots, N}$ (sinks). The characteristic matrix of $\pi$ is then the $N \times 2$-matrix

$$
C=\left(\begin{array}{cc}
\mathbb{1}_{S} & \mathbf{0}_{S} \\
\mathbf{0}_{N-S} & \mathbb{1}_{N-S}
\end{array}\right) .
$$


Then, computing $J_{\mathrm{PFE}}^{S} \mathbb{1}$, we find

$$
J_{\mathrm{PFE}}^{S} \mathbb{1}=\left(\begin{array}{c}
r_{s} \mathbb{1}_{S} \\
-r_{t} \mathbb{1}_{N-S}
\end{array}\right) .
$$

It follows that the column space of $C$ is $J_{\mathrm{PFE}}^{S}$-invariant, so by [15, Lemma 9.3.2], $\pi$ is equitable. As $\pi$ is equitable, it follows from [15, Theorem 9.3.1] that the quotient matrix $B_{\mathrm{PFE}}^{S}$ can be directly computed using the characteristic matrix $C$,

$$
B_{\mathrm{PFE}}^{S}=\left(C^{T} C\right)^{-1} C^{T} J_{\mathrm{PFE}}^{S} C .
$$

Thus,

$$
B_{\mathrm{PFE}}^{S}=\left(\begin{array}{cc}
m S-m N+r_{s} & m(N-S) \\
m S & -\left(m S+r_{t}\right)
\end{array}\right) .
$$

From [15, Theorem 9.3.3], the characteristic polynomial of $B_{\mathrm{PFE}}^{S}$ divides the characteristic polynomial of $J_{\mathrm{PFE}}^{S}$, i.e., $\sigma\left(B_{\mathrm{PFE}}^{S}\right) \subset \sigma\left(J_{\mathrm{PFE}}^{S}\right)$.

Furthermore, since $B_{\mathrm{PFE}}^{S}$ is essentially nonnegative, by the Perron-Frobenius Theorem, there exists a unique component-wise positive eigenvector $v_{p}$ such that $B_{\mathrm{PFE}}^{S} v_{p}=\lambda_{p} v_{p}=s\left(B_{\mathrm{PFE}}^{S}\right) v_{p}$. From [15, Theorem 9.3.1], we have that $J_{\mathrm{PFE}}^{S} C=C B_{\mathrm{PFE}}^{S}$. Thus,

$$
J_{\mathrm{PFE}}^{S} C v_{p}=C B_{\mathrm{PFE}}^{S} v_{p}=\lambda_{p} C v_{p}
$$

and therefore, $C v_{p}$ is an eigenvector of $J_{\mathrm{PFE}}^{S}$ that is also component-wise positive. Since the only component-wise positive eigenvector of $J_{\mathrm{PFE}}^{S}$ is the one corresponding to $s\left(J_{\mathrm{PFE}}^{S}\right)$, we have that $s\left(J_{\mathrm{PFE}}^{S}\right)=s\left(B_{\mathrm{PFE}}^{S}\right)$.

To compute $S^{c}$, recall that $S^{c}$ is the value of $S$ where the PFE loses local asymptotic stability, i.e., where $s\left(J_{\mathrm{PFE}}^{S}\right)=s\left(B_{\mathrm{PFE}}^{S}\right)=0$. So consider (19). We have $\operatorname{tr}\left(B_{\mathrm{PFE}}^{S}\right)=-m N+r_{s}-r_{t}$ and

$$
\operatorname{det}\left(B_{\mathrm{PFE}}^{S}\right)=-m S\left(r_{s}+r_{t}\right)-r_{s} r_{t}+m N r_{t} .
$$

If $r_{s}-r_{t}>m N$, then $\operatorname{tr}\left(B_{\mathrm{PFE}}^{S}\right)>0$ and necessarily the spectral abscissa of $B_{\mathrm{PFE}}^{S}$ is positive whenever $S \geq 1$. On the other hand $\operatorname{if} \operatorname{tr}\left(B_{\mathrm{PFE}}^{S}\right) \leq 0$ then the stability of $B_{\mathrm{PFE}}^{S}$ is governed by the determinant. In that case, the stability changes when

$$
S^{c}=\frac{m N r_{t}-r_{s} r_{t}}{m\left(r_{s}+r_{t}\right)}
$$

In the special case where $r_{s}=r_{t}=r$, we have

$$
S^{c}=\frac{N}{2}-\frac{r}{2 m} .
$$

\section{Numerical considerations}

We make some brief numerical remarks here. In what follows, matrix $\mathcal{M}$ is constructed as follows. First, we create a pattern matrix $\mathcal{M}_{p}$ that contains the 
digraph structure we want to impose: complete, path, ring, Barabasi-Albert, etc. We then create a values matrix $\mathcal{M}_{v}$ in which entries take values following some scheme explained later in specific examples. The movement matrix $\mathcal{M}$ itself is the Hadamard product $\mathcal{M}=\mathcal{M}_{p} \circ \mathcal{M}_{v}$ in which diagonal entries are then set to obtain the zero column sums property.

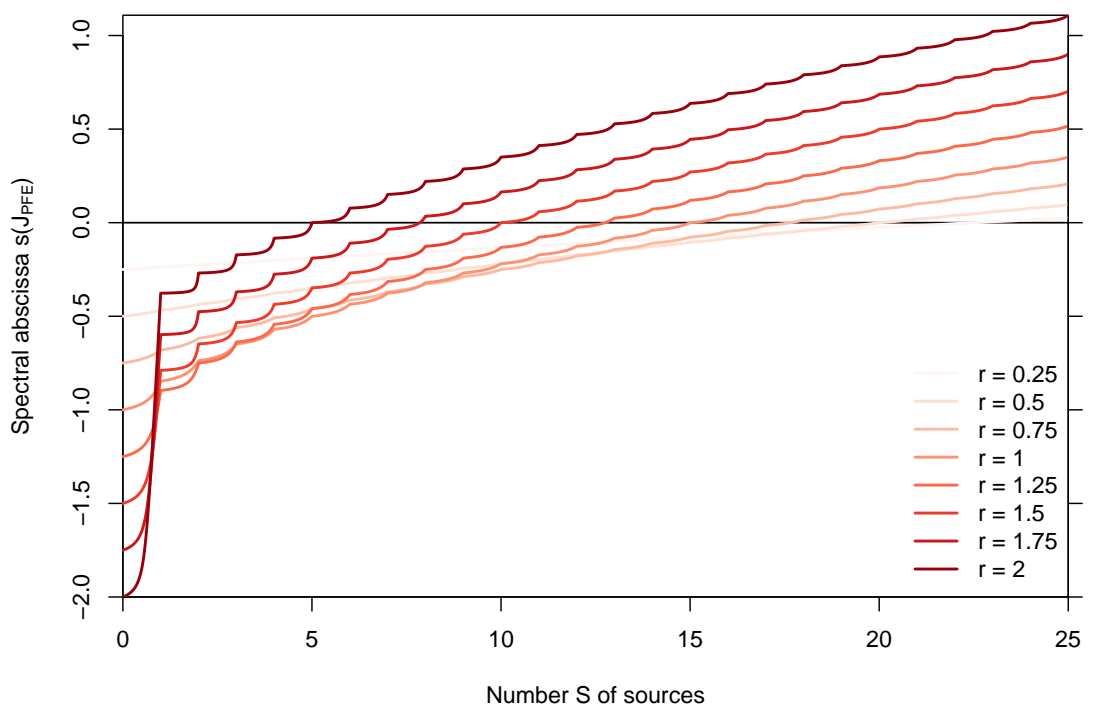

Figure 1: Situation that prevails when $N=50$ with a complete digraph of patches and movement rates taken all equal to 0.05 ; see text for details. Effect of varying $S \in[0, N]$ on the spectral abscissa $s\left(J_{\mathrm{PFE}}^{S}\right)$.

In Figure 1, the digraph is complete, making the movement matrix irreducible, entries in $\mathcal{M}_{v}$ all equal 0.05 and the total number of patches is $N=50$. The spectral abscissa $s\left(J_{\mathrm{PFE}}^{S}\right)$ of $J_{\mathrm{PFE}}^{S}$ as a function of $S$ is shown. We observe that while the PFE is initially stable when $S$ is small, as $S$ increases, there is a value $S^{c}$ of $S$ for which the PFE loses stability. As $r$ increases, that threshold $S^{c}$ decreases. The value of $S^{c}$ for a given curve is where the curve intersects the horizontal axis. Now focus on an individual curve in Figure 1 and observe that, as indicated by [10], perturbation of the diagonal leads to convex changes in the spectral abscissa on each sub-interval.

In Figure 2, we see the effect of increasing $S$ from 0 to 5 (with $N=50$ ), for several values of $r$, in the case of a Barabasi-Albert digraph, which is not strongly connected. Here, contrary to the case in Example 2, although the movement matrix is reducible, $\mathcal{S}_{\text {int }}$ is reduced to a single point for most values of $r$, although we do observe piecewise constant values of the spectral abscissa.

Notice that values of $S^{c}$ observed in Figure 2 are relatively small. This is due in part to the ordering of vertices used, as the $\mathrm{R}$ iGraph package used to generate the Barabasi-Albert digraph typically places vertex 1 at the "center". Although our mathematical results are established without loss of generality by 


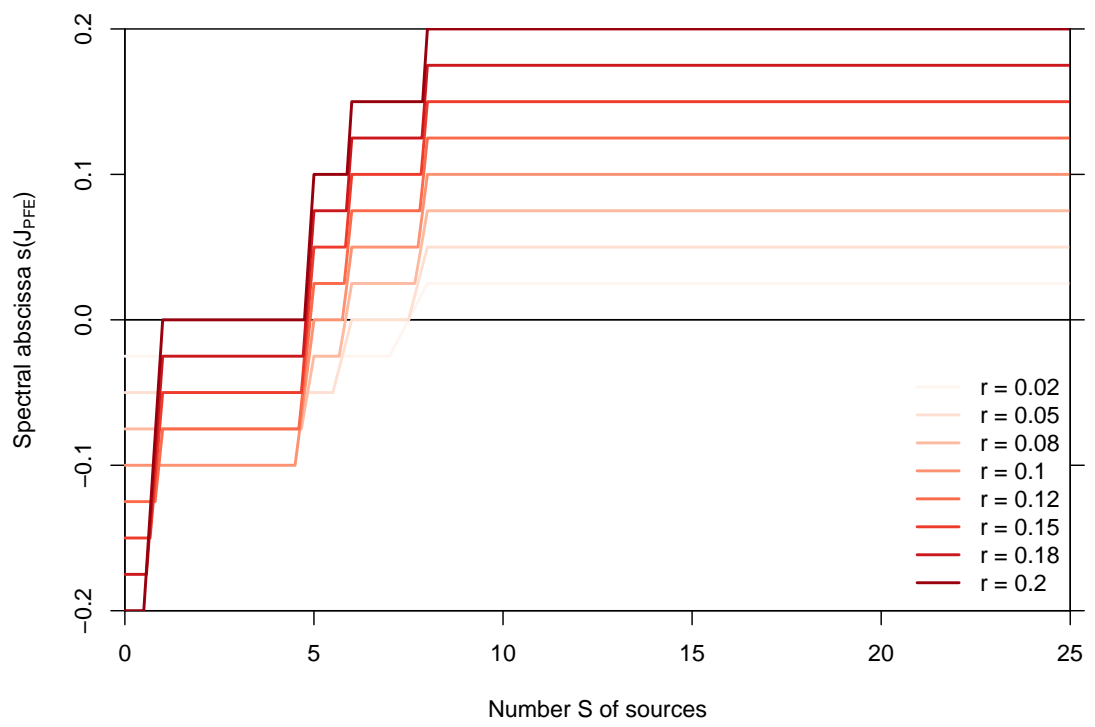

Figure 2: Effect of varying $S$ and $r$ on the spectral abscissa $s\left(J_{\mathrm{PFE}}^{S}\right)$. Here, the total number of patches is $N=50$ and $S$ varies between 0 and 5 . The underlying graph is an directed Barabasi-Albert graph.

arbitrarily ordering vertices, Example 1 shows that in practice, the nature of the graph influences $S^{c}$. In order to explore this in more detail, we proceed to a numerical experiment whose results are shown in Figure 3 . For legibility and computational efficiency, we pick two digraphs with $N=10$ vertices. The one in Figure 3a is of Barabasi-Albert type, while the one in Figure 3b is of Erdős-Rényi type. Nonzero movement rates are all taken as $m_{i j}=0.005$ and all $r_{i}=0.01$. For both digraphs, we then consider permutations of vertex labels, proceeding as follows. Denote $(1, \ldots, 10)$ the 10 -tuple of vertices for the digraphs shown in Figures $3 \mathrm{a}$ and $3 \mathrm{~b}$. Then, for instance, $(1, \ldots, 8,10,9)$ are digraphs obtained when the labels of vertices 9 and 10 are interchanged. Violin plots of values of $S^{c}$ when all $10 !=3,628,800$ such permutations are considered are shown in Figure 3c. Clearly, the structure of the connection digraph influences the range of values of $S^{c}$ and even, for a given digraph, so does the ordering of vertices.

Figure 4a shows the value of $S^{c}$ as a function of $m_{i j}$ and $r$. In this case, the digraph of patches is assumed to be complete (and therefore strongly connected, meaning the movement matrix is irreducible). The total number of patches is $N=50$. All movement rates are equal and this common value is varied on the $x$-axis, while the growth/death rate varies on the $y$-axis. Because movement rates are all equal and the movement matrix is irreducible, we are in the case of Section 3.6. The most favourable situation in terms of population survival thus happens when movement rates are low and growth/death rates are high. When movement rates are high, persistence of the population requires a very high birth/death rate or to have at least half of the patches as sources. 


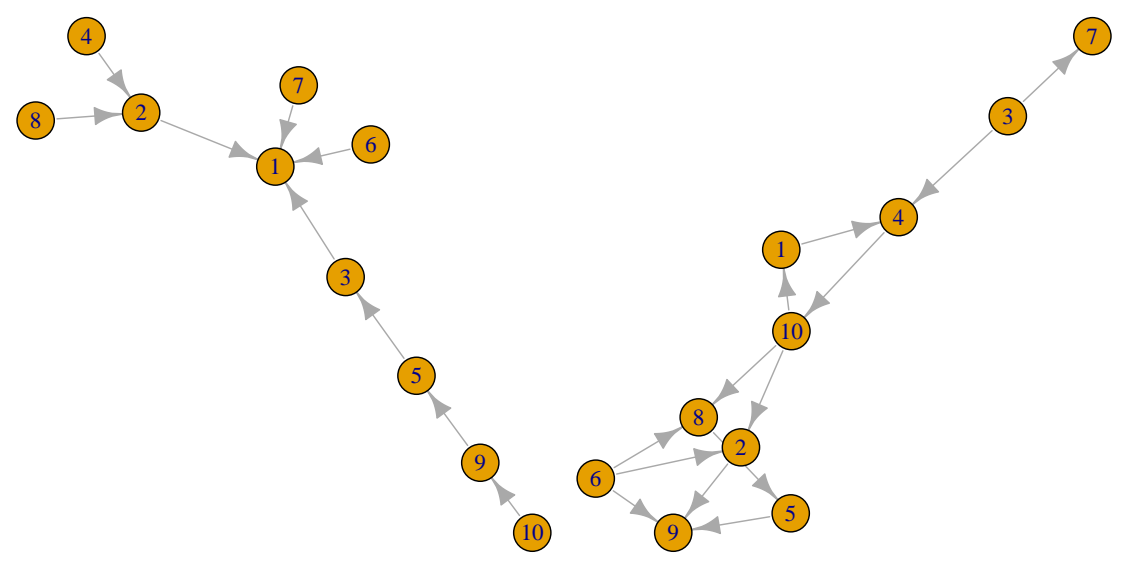

(a) Barabasi-Albert digraph

(b) Erdös-Rényi digraph

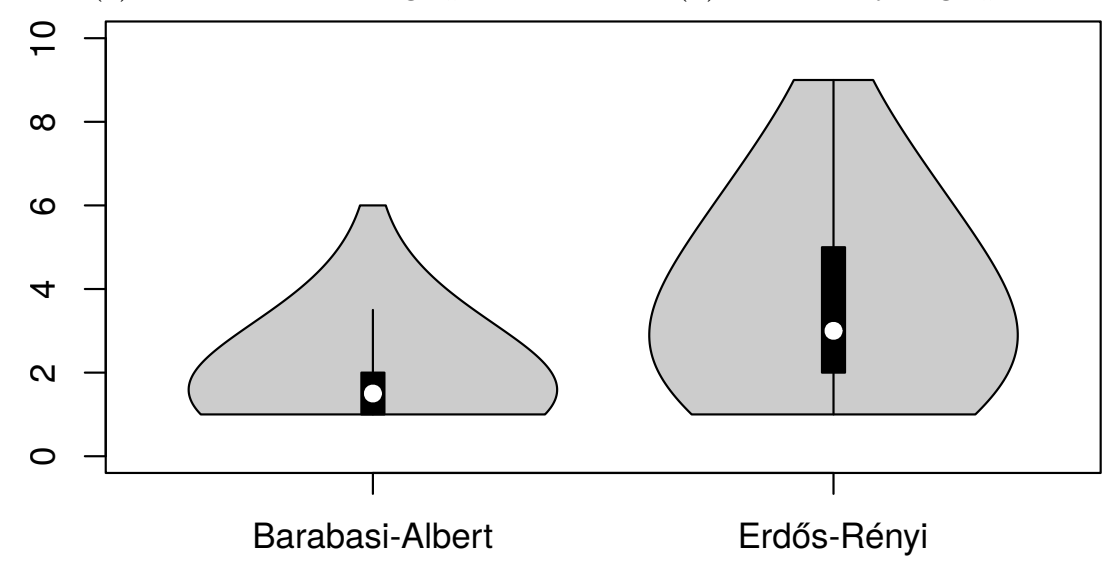

(c) Values of $S^{c}$

Figure 3: Two non-strongly connected 10-vertices digraphs of Barabasi-Albert (a) and Erdős-Rényi (b) type and violin plot of the corresponding ranges of values of $S^{c}$ when all permutations of vertices are considered (c); see text for details. All nonzero $m_{i j}=0.005$ and all $r_{i}=0.01$. In the violin plots, the black rectangle shows the interquartile range, the white dot within the black rectangle is the median and the light gray outer shape is a kernel density plot. 


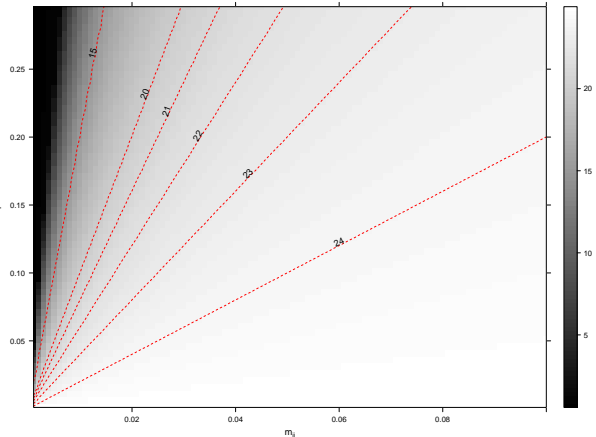

(a) Complete digraph

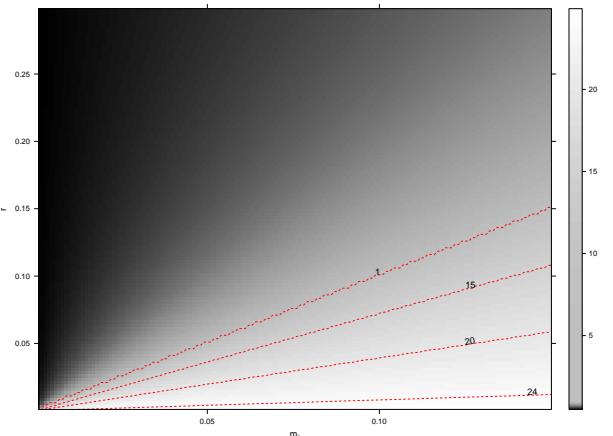

(b) One directional ring

Figure 4: Effect of variations of $m_{i j}$ and $r$ on the critical number $S^{c}$ in the case where $N=50$. (a) Complete digraph. (b) One-directional ring. Note that colour scales vary between the two figures.

Figure 4b, on the other hand, assumes that patches form a one-directional ring, i.e., $1 \rightarrow 2 \rightarrow \cdots \rightarrow N \rightarrow 1$. Because the movement digraph is not complete, the result of Section 3.6 does not hold. In this case, persistence of the population (i.e., existence of $P^{*} \gg 0$ ) is much more likely than in the case of Figure $4 \mathrm{a}$, since a large fraction of the $\left(m_{i j}, r\right)$ quadrant has $S^{c}<1$. Note that in both cases, we observe that $S^{c} \leq 25=N / 2$. This can be confirmed analytically in the case of Figure 4a, since in the limit, when $r=0$, it follows from (21) that $S^{c}=N / 2$. So in this case, population persistence is assured if over half of the patches are sources.

In all cases studied above, we have assumed that $r_{i}=r$ for all $i=1, \ldots, N$. To investigate the relative roles of the growth and death rates, let us now assume that $r_{i}=r_{s}, i=1, \ldots, S$ and $r_{i}=r_{t}, i=S+1, \ldots, N$. The same configuration as in Figure 4a is considered, with the $N=50$ patches forming a complete digraph. Movement rates are held equal at the value $m=0.01$. The result is shown in Figure 5. Here, $S^{c}$ can take values much larger than $N / 2$; for instance, the largest value of $S^{c}$ in Figure 5 is $\simeq 49.8$ and is reached when $\left(r_{s}, r_{t}\right)=(0.001,0.5)$. Indeed, with small values of $r_{s}$, it takes a lot of source patches in order for the population to persist. When $r_{t}$ is large, further changes to $r_{t}$ do not change the value of $S^{c}$ much; for very large values of $r_{t}$, the level lines of $S^{c}$ become essentially parallel to the $r_{t}$ axis (not shown). On the other hand, when $r_{t}$ is small, changes in $r_{t}$ have much more influence.

\section{Relation with existing work}

While the topic of persistence in metapopulations with explicit movement is quite novel in the sense it is approached here, there is a rich history of the topic in other contexts. We briefly present here some of these works, making the distinction between deterministic and stochastic approaches. Note that we 


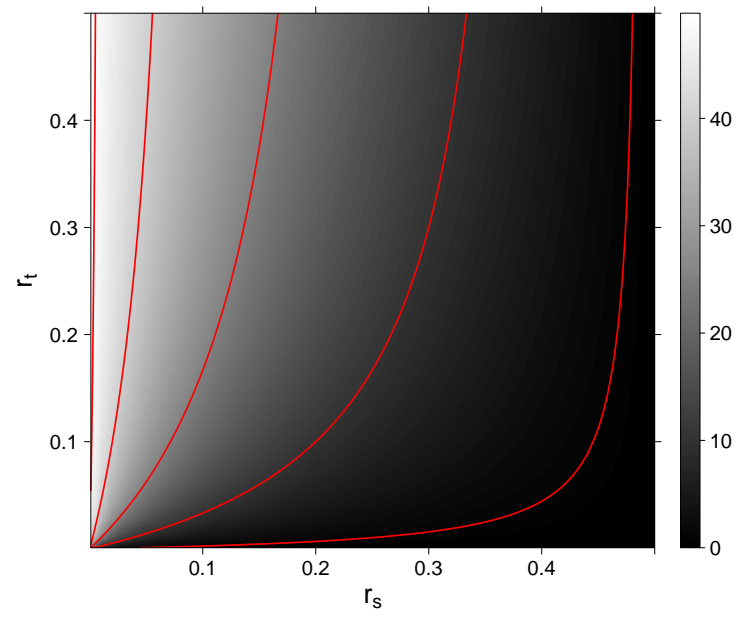

Figure 5: Effect of varying $r_{s}$ and $r_{t}$ on the spectral abscissa $s\left(J_{\mathrm{PFE}}^{S}\right)$, in the case of a complete digraph of $N=50$ patches. Red lines are located, from left to right, where $S^{c}$ equals $49,40,25,10$ and 1, respectively.

focus here on models for a single species; work is much more abundant for preypredator or competition models, but this is outside the scope of the present work.

\subsection{Deterministic approach}

Levins considers in [29] a metapopulation model in which the rate of change of the number of local populations $N$ is as follows

$$
N^{\prime}=\beta N\left(1-\frac{N}{T}\right)-\mu N,
$$

where $\beta$ is the immigration rate between patches, $T$ denotes the total number of patches and $\mu$ is the extinction rate of local populations.

Normalising (22) to have that $P$ is the fraction of occupied patches, [17] studies the following model:

$$
P^{\prime}=\beta P(h-P)-\mu P,
$$

where $h$ is the fraction of suitable patches. This model exhibits a threshold behaviour [17]: if $h<\mu / \beta$, then the PFE is globally asymptotically stable and if $h>\mu / \beta$, then there exists a unique nontrivial equilibrium $P^{*}$ that is globally asymptotically stable. Thus, the population persists if there are enough suitable patches; it goes extinct otherwise. The work [17] considers multiple variations on the above model. Of particular interest here is the following model for the (discrete) distributions $\mathbf{n}$ of patch qualities and $\mathbf{p}$ of number of occupied patches: 


$$
\begin{aligned}
& \mathbf{n}^{\prime}=\Gamma \mathbf{n} \\
& \mathbf{p}^{\prime}=C(t)(\mathbf{n}-\mathbf{p})-\mathcal{D} \mathbf{p}+\Gamma \mathbf{p},
\end{aligned}
$$

where $\Gamma$ is the matrix of rates of change in patch qualities, $\mathcal{D}=\left(\mu_{1}, \ldots, \mu_{m}\right)$ and

$$
C(t)=\sum_{i=1}^{m} \beta_{i} p_{i}(t)
$$

Note that $\Gamma$ has exactly the same structure as our movement matrix $\mathcal{M}$, although its interpretation is quite different. Clearly, if $\Gamma$ is irreducible, then $\mathbf{n} \rightarrow \mathbf{n}^{\star} \gg \mathbf{0}$ as $t \rightarrow \infty$, so (23) can be written as

$$
\mathbf{p}^{\prime}=C(t)\left(\mathbf{n}^{\star}-\mathbf{p}\right)-\mathcal{D} \mathbf{p}+\mathcal{M} \mathbf{p},
$$

so it is likely that with some adaptation, the method used here could be used on $(24)$.

Other notable work on the topic are $[18,19,21,26]$. Note that in the work of [17] as well as in most Levins-type metapopulations, the nontrivial equilibrium $P^{*}$ may be nonnegative. Thus, there can be persistence of the population overall without persistence of all local populations. This differs from our work, in which we find that there exists a strongly positive globally asymptotically stable equilibrium; see Theorem 1.

This difference is to a large extent due to the different scales of the problems being modelled by both types of approaches. Levins-type models assume that the number of patches is large enough that ordinary differential equations counting the number of patches in various states can be used. In this sense, they describe an ecosystem at the mesoscale. Metapopulation models with explicit movement, on the other hand, make an explicit description of each individual patch. As such, while they can be used for any number of patches, they are much more useful as a tool to consider properties on a smaller scale.

Metapopulation models with explicit movement are a little more recent than patch-occupancy models; they can be traced to the work of [28]. This was followed by some work in [20] and others. However, to the best of our knowledge, in discrete space with explicit population movement, only [2] provides a comparable result establishing the existence of a critical number of patches in order for the population to persist. (Most other works consider prey-predator or competition settings.) The model in [2] is obtained by discretising a one-dimensional reaction-diffusion equation. Adapting notation to ours here, it takes the form, for $i=1, \ldots, N$,

$$
P_{i}^{\prime}=f\left(P_{i}\right) P_{i}+m_{i, i+1}\left(P_{i+1}-P_{i}\right)+m_{i, i-1}\left(P_{i-1}-P_{i}\right) .
$$

In order to satisfy a zero Dirichlet boundary condition in the original partial differential equation, it is imposed that $P_{0}=P_{N+1}=0$. It is assumed that for 
all $P_{i}, \sup f\left(P_{i}\right)=r>0$, so all patches except for the extremities are, in effect, sources. Then [2] finds that the population goes extinct if

$$
r<2\left(\underline{m}+\bar{m} \cos \left(\frac{\pi N}{N+1}\right)\right),
$$

where $2 \underline{m}=\min _{i}\left\{m_{i, i+1}+m_{i, i-1}\right\}$ and $\bar{m}=\max _{i}\left\{m_{i, i+1}, m_{i, i-1}\right\}$. Also, there is a critical patch number $N_{c}$ such that $N<N_{c}$ implies that the population becomes extinct. Under specific model assumptions, $N_{c}$ can also be a sharp threshold, with the population becoming extinct for $N<N_{c}$ and persistent for $N>N_{c}$. Compare this with Theorem 1; there, if all patches are sources, then persistence is assured. The difference originates in the patches $P_{0}$ and $P_{N+1}$ used in [2]. Indeed, suppose that outflow towards these patches occurs at too high a rate. Then if the growth rate of the population in the system is not large enough, growth cannot compensate for this loss and the population goes extinct. As the model considered here is a closed system, this situation does not occur. We can however mimic the situation in [2] by considering patches in a line graph with the two extremities being sinks with $r_{i}$ values extremely large. In this case, we find (not shown) values of $S^{c}$ very close to $N$.

In [2], some considerations are also given about a more general model that uses the same hypotheses on the growth of populations but a more general movement network comparable to the one in the present paper. A thorough mathematical analysis of that model is conducted in [37].

\subsection{Stochastic approach}

Stochastic investigations have considered the effects of environmental stochasticity on metapopulation persistence. Using the concept of geometric mean fitness, many studies propose a persistence condition defined as a function of the geometric mean of growth rates [8, 27, 30,32]. Models used in these studies assume that dispersal occurs through individuals entering a common migrant pool. Early results propose to spread the risk arising from environmental stochasticity using a uniform dispersal in order to maximise the geometric mean [27]. More recently, $[7,8]$ propose the same modelling framework using a finite number of patches. They derive a critical number of patches below which the population goes extinct. Figure 2 in [8] and Figure 6 in [7] are similar to those obtained in the present study (Figures 1 and 2) and differ only in terms of the metric used.

In [13], it is observed that ideal-free dispersers occupy multiple patches in spatially heterogeneous environments provided environmental fluctuations are sufficiently strong and sufficiently weakly correlated across space. In contrast, for populations dispersing through diffusion and living in similar environments, intermediate dispersal rates maximise their stochastic growth rate. They also obtain an exact continuous counterpart to the critical number obtained in [8].

In [6], an individual-based model is used to study the respective effects of demography and dispersal when the environment is fluctuating. The authors propose a criterion for persistence, by studying the motion of a single random 
disperser until it returns to its initial position. They also provide a metric to assess the long term growth rate by using large deviations for the path of the random disperser. Such techniques provide criteria for persistence that depend on the demography of the population, as provided in the present study.

\section{Discussion}

The focus of this paper is the proof of existence of a threshold number $S^{c}$ of source patches in a metapopulation source-sink model, such that the populationfree equilibrium (PFE) is locally asymptotically stable below the threshold and unstable above it. In the case where the movement matrix is irreducible, we confirm that the threshold distinguishes between extinction of the population and its persistence at a positive globally asymptotically stable equilibrium. Furthermore, we provide an expression for the threshold $S^{c}$ when the movement digraph is complete and all movement rates are equal. Finally, some numerical investigations of the properties of the system are carried out.

Our model is a simplification of the model in [35]. Movement rates that we have denoted $m_{i j}$ here, take in [35] the form

$$
\frac{a_{i j}}{R_{i j}} \max \left\{0, p\left(P_{j}\right)-p\left(P_{i}\right)\right\},
$$

where $p\left(P_{i}\right)$ is the "pressure", defined as $p\left(P_{i}\right)=P_{i} / K_{i}$, and $R_{i j}$ is the "resistance" of the link $j \rightarrow i$, which the authors assume for instance to be a function of the distance between patches. Thus, in [35], at the PFE, there is no movement structure at all in the model. Because establishing a threshold requires working close to the PFE, we keep the formulation as in (1), which we consider to be the limit of the model in [35] when $P_{i} \rightarrow 0$. In the case of the model of [35], or in other source-sink systems where movement depends on population densities, e.g., $[3,34]$, the situation becomes more complex as one moves away from the population-free equilibrium.

The dynamical systems part of the analysis carried out here would not hold in some of the models where movement flow can be reversed by a change in the densities in origin and destination patches. In particular, the case where the underlying movement matrix is not pattern-symmetric, in the sense that $m_{i j} m_{j i}=0 \Longrightarrow m_{i j}=m_{j i}=0$, could generate more complicated dynamics and would be worthy of further investigation. In the case where the movement matrix is pattern symmetric, a reversal of the direction of flow is mitigated by the fact that it happens concurrently to an equivalent reversal along the reverse arc and thus only the cases where $m_{i j}$ and $m_{j i}$ vary widely are likely to generate more complicated dynamics. As previously mentioned, such flow reversals occur as one moves away from the PFE and thus the linear algebraic part of our arguments here would likely still hold. With some work, it should also be possible to extend the results here to cases where the dynamics in patches is more generic. 


\section{Acknowledgments}

JA and SK's work is supported in part by NSERC. NB was supported by INRIA. The authors acknowledge comments by Matts Gyllenberg that helped tie this work with existing literature.

\section{References}

[1] R. Agaev and P. Chebotarev. On the spectra of nonsymmetric Laplacian matrices. Linear Algebra and its Applications, 399:157-168, 2005.

[2] L.J.S. Allen. Persistence, extinction, and critical patch number for island populations. Journal of Mathematical Biology, 24(6):617-625, 1987.

[3] P. Amarasekare. The role of density-dependent dispersal in source-sink dynamics. Journal of Theoretical Biology, 226(2):159-168, 2004.

[4] R. Arditi, C. Lobry, and T. Sari. Is dispersal always beneficial to carrying capacity? New insights from the multi-patch logistic equation. Theoretical Population Biology, 106:45-59, 2015.

[5] J. Arino. Spatio-temporal spread of infectious pathogens of humans. Infectious Disease Modelling, 2:218-228, 2017.

[6] V. Bansaye and A. Lambert. New approaches to source-sink metapopulations decoupling demography and dispersal. Theoretical population biology, 88:31-46, 2013.

[7] J. Bascompte. Extinction thresholds: insights from simple models. In Annales Zoologici Fennici, pages 99-114. JSTOR, 2003.

[8] J. Bascompte, H. Possingham, and J. Roughgarden. Patchy populations in stochastic environments: critical number of patches for persistence. The American Naturalist, 159(2):128-137, 2002.

[9] A. Berman and R. J. Plemmons. Nonnegative Matrices in the Mathematical Sciences, volume 9 of Classics in Applied Mathematics. SIAM, 1994.

[10] E. Deutsch and M. Neumann. On the first and second order derivatives of the Perron vector. Linear Algebra and its Applications, 71:57-76, 1985.

[11] P.C. Dias. Sources and sinks in population biology. Trends in Ecology 83 Evolution, 11(8):326-330, 1996.

[12] O. Eriksson. Regional dynamics of plants: a review of evidence for remnant, source-sink and metapopulations. Oikos, pages 248-258, 1996.

[13] S.N. Evans, P.L. Ralph, S.J. Schreiber, and A. Sen. Stochastic population growth in spatially heterogeneous environments. Journal of mathematical biology, 66(3):423-476, 2013. 
[14] M. Fiedler. Special Matrices and their Applications in Numerical Mathematics. Dover, second edition, 2008.

[15] C. Godsil and G.F. Royle. Algebraic Graph Theory, volume 207 of Graduate Texts in Mathematics. Springer Science \& Business Media, 2013.

[16] D. Gravel, F. Guichard, M. Loreau, and N. Mouquet. Source and sink dynamics in meta-ecosystems. Ecology, 91(7):2172-2184, 2010.

[17] M. Gyllenberg and I. Hanski. Habitat deterioration, habitat destruction, and metapopulation persistence in a heterogenous landscape. Theoretical Population Biology, 52(3):198-215, 1997.

[18] I. Hanski. Single-species spatial dynamics may contribute to long-term rarity and commonness. Ecology, 66(2):335-343, 1985.

[19] I. Hanski. Single-species metapopulation dynamics: concepts, models and observations. Biological Journal of the Linnean Society, 42(1-2):17-38, 1991.

[20] A. Hastings. Dynamics of a single species in a spatially varying environment: the stabilizing role of high dispersal rates. Journal of Mathematical Biology, 16(1):49-55, 1982.

[21] A. Hastings. Structured models of metapopulation dynamics. Biological Journal of the Linnean Society, 42(1-2):57-71, 1991.

[22] M.W. Hirsch. The dynamical systems approach to differential equations. Bulletin of the American Mathematical Society, 11(1):1-64, 1984.

[23] R.D. Holt. Population dynamics in two-patch environments: some anomalous consequences of an optimal habitat distribution. Theoretical Population Biology, 28(2):181-208, 1985.

[24] R.A. Horn and C.R. Johnson. Topics in Matrix Analysis. Cambridge University Press, 1991.

[25] R.A. Horn and C.R. Johnson. Matrix Analysis. Cambridge University Press, 2nd edition, 2013.

[26] J.E. Keymer, P.A. Marquet, J.X. Velasco-Hernández, and S.A. Levin. Extinction thresholds and metapopulation persistence in dynamic landscapes. The American Naturalist, 156(5):478-494, 2000.

[27] E. Kuno. Dispersal and the persistence of populations in unstable habitats: a theoretical note. Oecologia, 49(1):123-126, 1981.

[28] S.A. Levin. Dispersion and population interactions. The American Naturalist, 108(960):207-228, 1974. 
[29] Richard Levins. Some demographic and genetic consequences of environmental heterogeneity for biological control. American Entomologist, 15(3):237-240, 1969.

[30] R.C. Lewontin and D. Cohen. On population growth in a randomly varying environment. Proceedings of the National Academy of sciences, 62(4):10561060, 1969.

[31] H. Matsumoto and H. Seno. On predator invasion into a multi-patchy environment of two kinds of patches. Ecological Modelling, 79(1):131 - 147, 1995.

[32] J.A.J. Metz, T.J. De Jong, and P.G.L. Klinkhamer. What are the advantages of dispersing; a paper by kuno explained and extended. Oecologia, 57(1-2):166-169, 1983.

[33] T. Namba. Dispersal-mediated coexistence of indirect competitors in source-sink metacommunities. Japan Journal of Industrial and Applied Mathematics, 24(1):39-55, Feb 2007.

[34] H.R. Pulliam. Sources, sinks, and population regulation. The American Naturalist, 132(5):652-661, 1988.

[35] R. Rael and C. Taylor. A flow network model for animal movement on a landscape with application to invasion. Theoretical Ecology, Apr 2018.

[36] H.L. Smith. Monotone dynamical systems, volume 41 of Mathematical Surveys and Monographs. American Mathematical Society, Providence, RI, 1995.

[37] Y. Takeuchi. Cooperative systems theory and global stability of diffusion models. Acta Applicandae Mathematicae, 14(1-2):49-57, 1989.

[38] E. Tromeur, L. Rudolf, and T. Gross. Impact of dispersal on the stability of metapopulations. Journal of Theoretical Biology, 392:1-11, 2016.

[39] R.S. Varga. Geršgorin and his Circles. Springer, 2010. 